Research Article

\title{
Hyers-Ulam-Rassias Stability of Additive Mappings in Fuzzy Normed Spaces
}

\author{
Jianrong Wu $\mathbb{D}$ and Lingxiao Lu \\ College of Mathematics Science, Suzhou University of Science and Technology, Suzhou, Jiangsu 215009, China \\ Correspondence should be addressed to Jianrong Wu; jrwu@mail.usts.edu.cn
}

Received 6 August 2021; Accepted 21 September 2021; Published 12 October 2021

Academic Editor: Ngai-Ching Wong

Copyright (C) 2021 Jianrong $\mathrm{Wu}$ and Lingxiao Lu. This is an open access article distributed under the Creative Commons Attribution License, which permits unrestricted use, distribution, and reproduction in any medium, provided the original work is properly cited.

In this paper, the Hyers-Ulam-Rassias stabilities of two functional equations, $f(a x+b y)=r f(x)+s f(y)$ and $f(x+y+z)=2 f((x+y) / 2)+f(z)$, are investigated in the framework of fuzzy normed spaces.

\section{Introduction}

The classical stability problem of functional equations is as follows: when is it true that a function which approximately satisfies a functional equation must be close to an exact solution of the equation? It originated from a question of Ulam [1] in 1940, concerning the stability of group homomorphisms. In the succeeding year, Hyers [2] gave a positive answer to the above question for additive groups under the assumption that the groups are Banach spaces. In 1978, Rassias [3] proved a generalization of Hyers' theorem for additive mappings. Since then, many papers and research monographs have been published on various generalizations and applications of the generalized Hyers-Ulam stability to a number of functional equations and mappings, such as the Cauchy equation $f(x+y)=f(x)+f(y)$, the Jensen equation $2 f((x+y) / 2)=f(x)+f(y)$, the quadratic equation, the cubic equation, and the mixed-type equation.

The reader is referred to [4-8] and references therein for detailed information on the stability of functional equations.

Studies on fuzzy normed spaces are relatively recent in the field of fuzzy functional analysis. In 1984, Katsaras [9] first introduced the idea of fuzzy norm on a linear space to construct a fuzzy vector topological structure on the space. After that, some mathematicians have defined fuzzy norms on a vector space from various points of view. In particular, Cheng and Mordeson [10] defined another type of fuzzy norm on a linear space in such a manner that the corresponding fuzzy metric is in the sense of Kramosil and Michalek [11].

With the development of fuzzy analysis, it has been involved in the Ulam stability. In 2008, Mirmostafaee and Moslehian [12] established some stability results concerning the cubic functional equation $f(2 x+y)+f$ $(2 x-y)=2 f(x+y)+2 f(x-y)+12 f(x)$ in fuzzy normed spaces. In the same year, Mirmostafaee et al. [13] proved a generalized Hyers-Ulam-Rassias stability theorem in the fuzzy sense.

Later, several various fuzzy versions of stability concerning Jensen, cubic, quadratic, and mixed additive-cubic functional equations were investigated (see, e.g., [14-24] and the references therein).

The main purpose of this paper is to establish the stability results concerning the following functional equations:

$$
f(a x+b y)=r f(x)+s f(y),
$$

where constants $a, b>0$ and $r, s \in R$ with $a+b=r+s \neq 1$, and

$$
f(x+y+z)=2 f\left(\frac{x+y}{2}\right)+f(z)
$$

in the setting of fuzzy normed spaces introduced in [10]. The achieved results via this paper improve and extend some recent well-known pertinent results. 
The organization of the paper is as follows: Section 2 comprises the basic notions on fuzzy normed spaces and some preliminary results. In Section 3, we prove Theorems 1 and 2 to show the stability of Cauchy equation (1) and Jensen equation (2), respectively. Finally, in Section 4, we give a brief conclusion.

In this paper, $\mathbb{R}$ and $\mathbb{N}$ stand for the set of all real numbers and the set of all positive integers, respectively.

\section{Terminology and Basic Notions}

In this section, we recall some necessary notions and fundamental results which are useful in this paper.

Definition 1 (see [10]). A fuzzy norm on a real vector space $X$ is a fuzzy set $N$ in $X \times[0, \infty)$ satisfying the following conditions for all $x, y \in X$ :

(FN1) $N(x, 0)=0$

(FN2) $N(x, t)=1$ for all $t>0$ if and only if $x=0$

(FN3) $N(c x, t)=N(x,(t /|c|))$ for all $t>0$ and $c \in \mathbb{R} \backslash\{0\}$

(FN4) $\min \{N(x, t), N(y, s)\} \leq N(x+y, t+s)$ for all $s, t \geq 0$

(FN5) $\lim _{t \rightarrow \infty} N(x, t)=1$

(FN6) $N(x, \cdot):[0, \infty) \longrightarrow[0,1]$ is left continuous

Let $(X, N)$ be a fuzzy normed vector space. A sequence $\left\{x_{n}\right\}$ in $X$ is said to be convergent or said to converge if there exists an $x \in X$ such that $\lim _{n \rightarrow \infty} N\left(x_{n}-x, t\right)=1$ for all $t>0$. In this case, $x$ is called the limit of the sequence $\left\{x_{n}\right\}$ and is denoted by $N-\lim _{n \rightarrow \infty} x_{n}=x$.

Remark 1. If $N-\lim _{n \rightarrow \infty} x_{n}=x$, then $N-\lim _{n \rightarrow \infty} \lambda x_{n}=$ $\lambda x$ for any $\lambda \in \mathbb{R}$.

A sequence $\left\{x_{n}\right\}$ in $X$ is said to be Cauchy if for each $\varepsilon>0$ and each $\delta>0$, there exists an $n_{0} \in \mathbb{N}$ such that $N\left(x_{m}-\right.$ $\left.x_{n}, \delta\right)>1-\varepsilon\left(m, n \geq n_{0}\right)$.

It is well known that every convergent sequence in a fuzzy normed space is Cauchy. If each Cauchy sequence is convergent, then the fuzzy norm is said to be complete and the fuzzy normed space is called a fuzzy Banach space.

Example 1. Let $(X$,$) be a normed vector space. Then,$

$$
N(x, t)= \begin{cases}0, & t=0, \\ \frac{t}{t+\|x\|}, & t>0\end{cases}
$$

is a fuzzy norm on $X$, and it is called the standard fuzzy norm on $(X$,$) . It is obvious that (X$,$) is complete if (X$,$) is$ complete.

\section{Stability of Functional Equations (1) and (2)}

Throughout this section, we assume that $X$ is a real vector space, the pair $(Y, N)$ is a fuzzy Banach space, and $\left(Z, N^{\prime}\right)$ is a fuzzy normed space.
First, we show the stability of functional equation (1). For notational convenience, given a mapping $f: X \longrightarrow Y$ of (1), we define a difference operator $D f: X^{2} \longrightarrow Y$ by

$D f(x, y)=f(a x+b y)-r f(x)-s f(y), \quad$ for all $x, y \in X$.

Theorem 1. Suppose $f: X \longrightarrow Y$ satisfies the inequality

$$
N(D f(x, y), t) \geq N^{\prime}(\varphi(x, y), t),
$$

for all $x, y \in X, t>0$, where constants $a, b>0$ and $r, s \in \mathbb{R}$ with $a+b=r+s \neq 1 ; \varphi: X^{2} \longrightarrow Z$ is a mapping for which there is a constant $0<\alpha<a+b$ such that

$$
N^{\prime}(\varphi((a+b) x,(a+b) x), t) \geq N^{\prime}(\alpha \varphi(x, x), t)
$$

and $\lim _{n \longrightarrow \infty} N^{\prime}\left(\varphi\left((a+b)^{n} x,(a+b)^{n} y\right),(a+b)^{n} t\right)=1$ for all $x, y \in X$. Then, one can find a unique additive mapping $T: X \longrightarrow Y$ satisfying the equation $D T(x, y)=0$ and the inequality

$$
\begin{aligned}
& N(T(x)-f(x), t) \\
& \quad \geq N^{\prime}\left(\varphi(x, x), \frac{(a+b-\alpha) t}{2}\right) .
\end{aligned}
$$

Proof. Letting $y=x$ in (5), we obtain

$$
\begin{aligned}
& N(f((a+b) x)-(r+s) f(x), t) \\
& \quad \geq N^{\prime}(\varphi(x, x), t) .
\end{aligned}
$$

Replacing $x$ by $(a+b)^{n} x(n \in \mathbb{N})$ in (8), we obtain

$$
\begin{aligned}
& N\left(f\left((a+b)^{n+1} x\right)-(r+s) f\left((a+b)^{n} x\right), t\right) \\
& \quad \geq N^{\prime}\left(\varphi\left((a+b)^{n} x,(a+b)^{n} x\right), t\right) .
\end{aligned}
$$

This together with (6) implies that

$$
\begin{aligned}
& N\left(\frac{f\left((a+b)^{n+1} x\right)}{(a+b)^{n+1}}-\frac{f\left((a+b)^{n} x\right)}{(a+b)^{n}}, \frac{t}{(a+b)^{n+1}}\right) \\
& \quad \geq N^{\prime}\left(\varphi\left((a+b)^{n} x,(a+b)^{n} x\right), t\right) \\
& \quad \geq N^{\prime}\left(\alpha^{n} \varphi(x, x), t\right)=N^{\prime}\left(\varphi(x, x), \frac{t}{\alpha^{n}}\right) .
\end{aligned}
$$

By replacing $t$ with $\alpha^{n} t$, we obtain

$$
\begin{aligned}
& N\left(\frac{f\left((a+b)^{n+1} x\right)}{(a+b)^{n+1}}-\frac{f\left((a+b)^{n} x\right)}{(a+b)^{n}}, \frac{\alpha^{n} t}{(a+b)^{n+1}}\right) \\
& \quad \geq N^{\prime}(\varphi(x, x), t) .
\end{aligned}
$$

Then, 


$$
\begin{aligned}
& N\left(\frac{f\left((a+b)^{n+p} x\right)}{(a+b)^{n+p}}-\frac{f\left((a+b)^{n} x\right)}{(a+b)^{n}}, \sum_{i=n}^{n+p-1} \frac{\alpha^{i} t}{(a+b)^{i+1}}\right) \\
& \quad=N\left(\sum_{i=n}^{n+p-1}\left(\frac{f\left((a+b)^{i+1} x\right)}{(a+b)^{i+1}}-\frac{f\left((a+b)^{i} x\right)}{(a+b)^{i}}\right), \sum_{i=n}^{n+p-1} \frac{\alpha^{i} t}{(a+b)^{i+1}}\right) \\
& \quad \geq \min _{n \leq i \leq n+p-1} N\left(\frac{f\left((a+b)^{i+1} x\right)}{(a+b)^{i+1}}-\frac{f\left((a+b)^{i} x\right)}{(a+b)^{i}}, \frac{\alpha^{i} t}{(a+b)^{i+1}}\right) \\
& \geq N^{\prime}(\varphi(x, x), t) .
\end{aligned}
$$

Hence,

$$
\begin{aligned}
& N\left(\frac{f\left((a+b)^{n+p} x\right)}{(a+b)^{n+p}}-\frac{f\left((a+b)^{n} x\right)}{(a+b)^{n}}, t\right) \\
& \quad \geq N^{\prime}\left(\varphi(x, x), \frac{t}{\sum_{i=n}^{n+p-1} \alpha^{i} /(a+b)^{i+1}}\right) .
\end{aligned}
$$

Since $\sum_{k=0}^{\infty}\left(\alpha^{k} /(a+b)^{k+1}\right)=(1 /(a+b-\alpha))<\infty$, the Cauchy criterion for convergence shows that $\left\{f_{n}(x)\right\}$ is a Cauchy sequence in $(Y, N)$, where $f_{0}(x)=f(x)$ and $f_{n}(x)=f\left((a+b)^{n} x\right) /(a+b)^{n} \quad(n \in \mathbb{N})$. Thanks to $(Y, N)$ being a fuzzy Banach space, this sequence converges to a point $T(x) \in Y$. That is, $T(x)=N-\lim _{n \longrightarrow \infty} f_{n}(x)$.

From (11), we know that

$$
\begin{aligned}
& N\left(f_{n}(x)-f(x), \sum_{k=0}^{n-1} \frac{\alpha^{k} t}{(a+b)^{k+1}}\right) \\
& \quad=N\left(\sum_{k=0}^{n-1}\left(f_{k+1}(x)-f_{k}(x)\right), \sum_{k=0}^{n-1} \frac{\alpha^{k} t}{(a+b)^{k+1}}\right) \\
& \geq \min _{0 \leq k \leq n-1}\left\{N\left(f_{k+1}(x)-f_{k}(x), \frac{\alpha^{k} t}{(a+b)^{k+1}}\right)\right\} \\
& \geq N^{\prime}(\varphi(x, x), t) .
\end{aligned}
$$

Therefore,

$$
\begin{aligned}
& N\left(f_{n}(x)-f(x), t\right) \\
& \quad \geq N^{\prime}\left(\varphi(x, x), \frac{t}{\sum_{k=0}^{n-1} \alpha^{k} /(a+b)^{k+1}}\right) \\
& \quad \geq N^{\prime}\left(\varphi(x, x), \frac{t}{\sum_{k=0}^{\infty} \alpha^{k} /(a+b)^{k+1}}\right) \\
& \quad=N^{\prime}(\varphi(x, x),(a+b-\alpha) t) .
\end{aligned}
$$

Hence,

$$
\begin{aligned}
N & (T(x)-f(x), t) \\
& \geq \min \left\{N\left(T(x)-f_{n}(x), \frac{t}{2}\right), N\left(f_{n}(x)-f(x), \frac{t}{2}\right)\right\} \\
& \geq N^{\prime}\left(\varphi(x, x), \frac{(a+b-\alpha) t}{2}\right),
\end{aligned}
$$

for $n$ large enough.

Now, we prove $T$ is an additive mapping. Replace $x$ and $y$ by $(a+b)^{n} x$ and $(a+b)^{n} y$, respectively, in (5) to obtain

$$
\begin{aligned}
& N\left(\frac{f\left((a+b)^{n}(a x+b y)\right.}{(a+b)^{n}}-\frac{r f\left((a+b)^{n} x\right)}{(a+b)^{n}}-\frac{s f\left((a+b)^{n} y\right)}{(a+b)^{n}}, t\right. \\
& \quad \geq N^{\prime}\left(\varphi\left((a+b)^{n} x,(a+b)^{n} y\right),(a+b)^{n} t\right),
\end{aligned}
$$

for all $x, y \in X$ and all $t>0$. Since $\lim _{n \rightarrow \infty} N^{\prime}\left(\varphi\left((a+b)^{n} x,(a+b)^{n} y\right),(a+b)^{n} t\right)=1$, we conclude that $\operatorname{DT}(x, y)=0$.

To prove the uniqueness of the additive mapping $T$, assume that there exists another additive mapping $S: X \longrightarrow Y$ which satisfies (7). Since DT $(x, y)=0$, we have $T(a x+b y)=r T(x)+s T(y)$. Let $x=y$, and then,

$$
T((a+b) x)=(r+s) T(x)=(a+b) T(x) .
$$

Consequently, $T\left((a+b)^{k} x\right)=(a+b)^{k} T(x)$. Similarly, $S\left((a+b)^{k} x\right)=(a+b)^{k} S(x)$ for all $x \in X$ and $k \in \mathbb{N}$. From (7) and (6), we have

$$
\begin{aligned}
N & (T(x)-S(x), t) \\
& =N\left(T\left((a+b)^{n} x\right)-S\left((a+b)^{n} x\right),(a+b)^{n} t\right) \\
& \geq \min \left\{N\left(T\left((a+b)^{n} x\right)-f\left((a+b)^{n} x\right), \frac{(a+b)^{n} t}{2}\right),\right.
\end{aligned}
$$




$$
\begin{aligned}
& \left.N\left(f\left((a+b)^{n} x\right)-S\left((a+b)^{n} x\right), \frac{(a+b)^{n} t}{2}\right)\right\} \\
& \quad \geq N^{\prime}\left(\varphi\left((a+b)^{n} x,(a+b)^{n} x\right),\right) \\
& \quad \geq N^{\prime}\left(\varphi(x, x), \frac{(a+b-\alpha)(a+b)^{n} t}{4 \alpha^{n}}\right) .
\end{aligned}
$$

Since $\lim _{n \rightarrow \infty}\left(\left((a+b-\alpha)(a+b)^{n} t\right) / 4 \alpha^{n}\right)=\infty$, we obtain $N(T(x)-S(x), t)=1$ for all $t>0$. Therefore, $T(x)-S(x)=0$. The proof ends.

Example 2. Let $X$ be a Banach space and $Z$ be a normed space. Denote by $N$ and $N^{\prime}$ the standard fuzzy norms on $X$ and $Z$, respectively. Then, $(X, N)$ is a fuzzy Banach space. Let $\varphi: X^{2} \longrightarrow Z$ be denoted by

$$
\varphi(x, y)=\left(\frac{\|x\|^{2}}{2}+\frac{\|y\|^{2}}{6}\right) z_{0}
$$

where $z_{0}$ is a fixed unit vector in $Z$. Define $f: X \longrightarrow X$ by

$$
f(x)=x+\|x\|^{2} x_{0}
$$

where $x_{0}$ is a fixed unit vector in $X$. Set $a=b=1 / 3, s=1 / 6$, and $r=1 / 2$; then, $a+b=r+s \neq 1$ and

$$
\begin{array}{r}
D f(x, y)=f(a x+b y)-r f(x)-s f(y) \\
=\left(\frac{\|x+y\|}{9}-\frac{\|x\|}{2}-\frac{\|y\|}{6}\right) x_{0} .
\end{array}
$$

Therefore,

$$
\begin{aligned}
N & (D f(x, y), t) \\
& =\frac{t}{t+\left\|((\|x+y\| / 9)-(\|x\| / 2)-(\|y\| / 6)) x_{0}\right\|} \\
& =\frac{18 t}{18 t+9\|x\|^{2}-2\|x+y\|^{2}+3\|y\|^{2}} \\
& \geq \frac{18 t}{18 t+9\|x\|^{2}+3\|y\|^{2}} \\
& =N^{\prime}(\varphi(x, y), t), \\
N^{\prime} & (\varphi((a+b) x,(a+b) x), t) \\
& =\frac{t}{t+\|\varphi((2 x / 3),(2 x / 3))\|} \\
& =N^{\prime}(\alpha \varphi(x, x), t), \\
& =\frac{t}{t+\alpha\|\varphi(x, x)\|}
\end{aligned}
$$

where $\alpha=(4 / 9)<(2 / 3)=a+b$. Moreover,

$$
\begin{gathered}
\lim _{n \longrightarrow \infty} N^{\prime}\left(\varphi\left((a+b)^{n} x,(a+b)^{n} y\right),(a+b)^{n} t\right) \\
\quad=\lim _{n \longrightarrow \infty} N^{\prime}\left(\varphi\left(\left(\frac{2}{3}\right)^{n} x,\left(\frac{2}{3}\right)^{n} y\right),\left(\frac{2}{3}\right)^{n} t\right) \\
\quad=\lim _{n \longrightarrow \infty} \frac{(2 / 3)^{n} t}{(2 / 3)^{n} t+(2 / 3)^{2 n}\|\varphi(x, y)\|}=1 .
\end{gathered}
$$

Hence, the conditions of Theorem 1 are fulfilled. Therefore, there is a unique additive mapping $T: X \longrightarrow X$ satisfying the inequality

$$
\begin{aligned}
N & (T(x)-f(x), t) \\
& \geq N^{\prime}\left(\varphi(x, x), \frac{(a+b-\alpha) t}{2}\right) \\
& =N^{\prime}\left(\frac{4}{6}\|x\|^{2}, \frac{t}{9}\right) .
\end{aligned}
$$

Indeed,

$$
\begin{aligned}
T(x) & =N-\lim _{n \rightarrow \infty} f_{n}(x) \\
& =N-\lim _{n \longrightarrow \infty} \frac{f\left((2 / 3)^{n} x\right)}{(2 / 3)^{n}} \\
& =N-\lim _{n \longrightarrow \infty} \frac{(2 / 3)^{n} x+\left\|(2 / 3)^{n} x\right\|^{2} x_{0}}{(2 / 3)^{n}} \\
& =N-\lim _{n \rightarrow \infty}\left(x+\left(\frac{2}{3}\right)^{n}\|x\|^{2} x_{0}\right) \\
& =x, \quad \forall x \in X .
\end{aligned}
$$

Now, we show the stability of functional equation (2). For the mapping $f: X \longrightarrow Y$ of (2), we define a difference operator $D f: X^{3} \longrightarrow Y$ by

$$
\begin{aligned}
D f & (x, y, z) \\
& =f(x+y+z)-2 f\left(\frac{x+y}{2}\right)-f(z) .
\end{aligned}
$$

Theorem 2. Let $\varphi: X^{3} \longrightarrow Z$ be a mapping for which there is a constant $0<\alpha<3$, such that

$$
\begin{aligned}
& N^{\prime}(\varphi(3 x, 3 x, 3 x), t) \\
& \quad \geq N^{\prime}(\alpha \varphi(x, x, x), t),
\end{aligned}
$$

and $\lim _{n \rightarrow \infty} N^{\prime}\left(\varphi\left(3^{n} x, 3^{n} y, 3^{n} z\right), 3^{n} t\right)=1$ for all $x, y, z \in X$. Assume that a mapping $f: X \longrightarrow Y$ satisfies the inequality

$$
N(D f(x, y, z), t) \geq N^{\prime}(\varphi(x, y, z), t)
$$

for all $x, y, z \in X$ and $t>0$. Then, one can find a unique additive mapping $T: X \longrightarrow Y$ satisfying the equation $\operatorname{DT}(x, y, z)=0$ and the inequality 


$$
\begin{aligned}
& N(T(x)-f(x), t) \\
& \quad \geq N^{\prime}\left(\varphi(x, x, x), \frac{(3-\alpha) t}{6}\right) .
\end{aligned}
$$

Proof. The techniques are completely similar to that of Theorem 1. Hence, we present a sketch of proof. Put $x=$ $y=z$ in (29) to obtain

$$
\begin{array}{r}
N(f(3 x)-3 f(x), t) \\
\geq N^{\prime}(\varphi(x, x, x), t) .
\end{array}
$$

Replacing $x$ and $t$ by $3^{n} x$ and $\alpha^{n} t$, respectively, we obtain

$$
\begin{aligned}
& N\left(\frac{f\left(3^{n+1} x\right)}{3^{n+1}}-\frac{f\left(3^{n} x\right)}{3^{n}}, \frac{\alpha^{n} t}{3^{n}}\right) \\
& \quad \geq N^{\prime}(\varphi(x, x, x), t) .
\end{aligned}
$$

Denote $f_{0}(x)=f(x)$ and $f_{n}(x)=f\left(3^{n} x\right) / 3^{n}$, where $x \in X$ and $n \in \mathbb{N}$; we know

$$
\begin{aligned}
& N\left(f_{n+1}(x)-f_{n}(x), \frac{\alpha^{n} t}{3^{n}}\right) \\
& \geq N^{\prime}(\varphi(x, x, x), t) .
\end{aligned}
$$

Then,

$$
\begin{aligned}
& N\left(f_{n+p}(x)-f_{n}(x), \sum_{i=n}^{n+p-1} \frac{\alpha^{i} t}{3^{i}}\right) \\
& \geq N^{\prime}(\varphi(x, x, x), t) .
\end{aligned}
$$

Hence,

$$
\begin{aligned}
& N\left(f_{n+p}(x)-f_{n}(x), t\right) \\
& \quad \geq N^{\prime}\left(\varphi(x, x, x), \frac{t}{\sum_{i=n}^{n+p-1} \alpha^{i} / 3^{i}}\right) .
\end{aligned}
$$

Since $\sum_{k=0}^{\infty}\left(\alpha^{k} / 3^{k}\right)=(3 /(3-\alpha))<\infty, N-\lim _{n \longrightarrow \infty} f_{n}$ $(x)=T(x) \in Y$.

It follows from (33) that

$$
\begin{aligned}
& N\left(f_{n}(x)-f(x), \sum_{k=0}^{n-1} \frac{\alpha^{k} t}{3^{k}}\right) \\
& \geq N^{\prime}(\varphi(x, x, x), t) .
\end{aligned}
$$

Therefore,

$$
\begin{aligned}
& N\left(f_{n}(x)-f(x), t\right) \\
& \quad \geq N^{\prime}\left(\varphi(x, x, x), \frac{(3-\alpha) t}{3}\right) .
\end{aligned}
$$

Hence,

$$
\begin{aligned}
N & (T(x)-f(x), t) \\
& \geq \min \left\{N\left(T(x)-f_{n}(x), \frac{t}{2}\right), N\left(f_{n}(x)-f(x), \frac{t}{2}\right)\right\} \\
& \geq N^{\prime}\left(\varphi(x, x, x), \frac{(3-\alpha) t}{6}\right),
\end{aligned}
$$

for $n$ large enough.

To prove $T$ is an additive mapping, we replace $x$ and $y$ by $3^{n} x$ and $3^{n} y$, respectively, in (29), and we obtain

$$
\begin{aligned}
& N\left(\frac{f\left(3^{n} x+3^{n} y+3^{n} z\right)}{3^{n}}\right. \\
& \left.\quad-\frac{2}{3^{n}} f\left(\frac{3^{n} x+3^{n} y}{2}\right)-\frac{1}{3^{n}} f\left(3^{n} z\right), t\right) \\
& \quad \geq N^{\prime}\left(\varphi\left(3^{n} x, 3^{n} y, 3^{n} z\right), 3^{n} t\right),
\end{aligned}
$$

for all $x, y, z \in X$ and all $t>0$. Since $\lim _{n \rightarrow \infty} N^{\prime}\left(\varphi\left(3^{n} x, 3^{n} y, 3^{n} z\right), 3^{n} t\right)=1$, we conclude that $\operatorname{DT}(x, y, z)=0$.

To prove the uniqueness of the additive mapping $T$, assume that there exists another additive mapping $S: X \longrightarrow Y$ which satisfies (30). It is easy to see that $T\left(3^{k} x\right)=3^{k} T(x)$ and $S\left(3^{k} x\right)=3^{k} S(x)$ for all $x \in X$ and $k \in \mathbb{N}$. This together with (30) and (28) implies that

$$
\begin{aligned}
& N(T(x)-S(x), t) \\
& \quad \geq N^{\prime}\left(\varphi(x, x, x), \frac{(3-\alpha) 3^{n} t}{12 \alpha^{n}}\right) .
\end{aligned}
$$

Since $0<\alpha<3$, we obtain $N(T(x)-D(x), t)=1$ for all $t>0$. Therefore, $T(x)-S(x)=0$. The proof ends.

\section{Conclusion}

By using the direct method, we establish the Hyers-Ulam-Rassias stability of the functional equations in the framework of fuzzy normed spaces. We show that the existence and uniqueness of the solutions for these equations. These circumstances can be applied to other significant functional equations in the set of fuzzy normed spaces.

\section{Data Availability}

No data were used to support this study.

\section{Conflicts of Interest}

The authors declare no conflicts of interest.

\section{Authors' Contributions}

Conceptualization, funding acquisition, methodology, and reviewing and editing of the manuscript were performed by Jianrong $\mathrm{Wu}$. Formal analysis, investigation, and original 
draft preparation were carried out by Lingxiao Lu. Both authors have read and agreed to the published version of the manuscript.

\section{Acknowledgments}

The authors acknowledge the support of the National Natural Science Foundation of China (Grant no. 11971343).

\section{References}

[1] S. M. Ulam, Problems in Modern Mathematics, Wiley, New York, NY, USA, 1940.

[2] D. H. Hyers, "On the stability of the linear functional equation," Proceedings of the National Academy of Sciences, vol. 27 , no. 4 , pp. 222-224, 1941.

[3] T. M. Rassias, "On the stability of the linear mapping in Banach spaces," Proceedings of the American Mathematical Society, vol. 72, no. 2, pp. 297-300, 1978.

[4] N. Brillouët-Belluot, J. Brzdek, and K. Cieplinski, "On some recent developments in Ulam's type stability," Abstract and Applied Analysis, vol. 2012, Article ID 716936, 2012.

[5] I.-i. EL-Fassi, "On the general solution and hyperstability of the general radical quintic functional equation in quasi$\beta$-Banach spaces," Journal of Mathematical Analysis and Applications, vol. 466, no. 1, pp. 733-748, 2018.

[6] I. Hwang and C. Park, "Ulam stability of an additive-quadratic functional equation in Banach spaces," Journal of Mathematical Inequalities, vol. 2020, no. 2, pp. 421-436, 2020.

[7] S.-S. Jin and Y.-H. Lee, "On the Hyers-Ulam-Rassias stability of an additive-cubic functional equation," International Journal of Mathematical Analysis, vol. 13, no. 5, pp. 213-221, 2019.

[8] C. Park, S. M. Kwon, and J. R. Lee, "Hyers-Ulam stability of additive function equations in paranormed spaces," Journal of Computational Analysis and Applications, vol. 26, no. 3, pp. 532-538, 2019.

[9] A. K. Katsaras, "Fuzzy topological vector spaces II," Fuzzy Sets and Systems, vol. 12, no. 2, pp. 143-154, 1984.

[10] S. C. Cheng and J. N. Mordeson, "Fuzzy linear operators and fuzzy normed linear spaces," Bulletin of the Calcutta Mathematical Society, vol. 86, no. 5, pp. 429-436, 1994.

[11] I. Kramosil and J. Michalek, "Fuzzy metrics and statistical metric spaces," Kybernetika, vol. 11, pp. 336-344, 1975.

[12] A. K. Mirmostafaee and M. S. Moslehian, "Fuzzy approximately cubic mappings," Information Sciences, vol. 178, no. 19, pp. 3791-3798, 2008.

[13] A. K. Mirmostafaee, M. Mirzavaziri, and M. S. Moslehian, "Fuzzy stability of the Jensen functional equation," Fuzzy Sets and Systems, vol. 159, no. 6, pp. 730-738, 2008.

[14] M. E. Gordji, H. Khodaei, and M. Kamyar, "Stability of Cauchy-Jensen type functional equation in generalized fuzzy normed spaces," Computers and Mathematics with Applications, vol. 62, no. 8, pp. 2950-2960, 2011.

[15] V. Govindan and S. Murthy, "Solution and Hyers-Ulam stability of $n$-dimensional non-quadratic functional equation in fuzzy normed space using direct method," Materials Today: Proceedings, vol. 16, pp. 384-391, 2019.

[16] S. Y. Jang and C. Park, "Fuzzy stability of a functional equation related to inner product spaces," Hackett University Bulletin of the Natural Sciences and Engineering, vol. 40, no. 5, pp. 711-723, 2014.

[17] H. A. Kenary and T. M. Rassias, "On the HUR-stability of quadratic functional equations in fuzzy Banach spaces,," in
Applications of Nonlinear Analysis. Springer Optimization and its Applications 134, T. M. Rassias, Ed., Springer, Basel, Switzerland, 2018.

[18] D. Miheţ, "The fixed point method for fuzzy stability of the Jensen functional equation," Fuzzy Sets and Systems, vol. 160, no. 11, pp. 1663-1667, 2009.

[19] A. K. Mirmostafaee, "A fixed point approach to almost quartic mappings in quasi fuzzy normed spaces," Fuzzy Sets and Systems, vol. 160, no. 11, pp. 1653-1662, 2009.

[20] E. Movahednia, S. Eshtehar, and Y. Son, "Stability of quadratic functional equations in fuzzy normed spaces," International Journal of Mathematics and Analysis, vol. 6, pp. 2405-2412, 2012.

[21] P. Narasimman, H. Dutta, and I. H. Jebril, "Stability of mixed type functional equation in normed spaces using fuzzy concept," International Journal of General Systems, vol. 2019, pp. 1-16, 2019.

[22] C. Park, "Fixed points and stability of the Cauchy-Jensen functional inequality in fuzzy Banach algebras," Applied Mathematics Letters, vol. 24, no. 12, pp. 2024-2029, 2011.

[23] C. Park, D. Shin, R. Saadati, and J. Lee, "A fixed point approach to the fuzzy stability of an AQCQ-functional equation," Filomat, vol. 30, no. 7, pp. 1833-1851, 2016.

[24] J. Shokri and C. Park, "A fixed point approach to approximate quadratic functional equation in non-Archimedean $L^{*}$-fuzzy normed spaces," Journal of Intelligent and Fuzzy Systems, vol. 38 , no. 6 , pp. $1-8,2020$. 